\title{
Medical Imaging Analysis with Artificial Neural Networks
}

\author{
J. Jiang, P. Trundle and J. Ren \\ Digital Media \& Systems Research Institute, University of Bradford, \\ Bradford, BD7 1DP, United Kingdom \\ Email: (j.jiang1, p.r.trundle, j.ren)@bradford.ac.uk
}

\begin{abstract}
Given the fact that neural networks have been widely reported in the research community of medical imaging, we provide a focused literature survey on recent neural network developments in computeraided diagnosis, medical image segmentation and edge detection toward visual content analysis, and medical image registration for its pre-processing and post processing, with the aim of increasing awareness of how neural networks can be applied to these areas and providing a foundation for further research and practical development. Representative techniques and algorithms are explained in detail to provide inspiring examples illustrating: (i) how a known neural network with fixed structure and training procedure could be applied to resolve a medical imaging problem; (ii) how medical images could be analysed, processed, and characterised by neural networks; and (iii) how neural networks could be expanded further to resolve problems relevant to medical imaging. In the concluding section, a highlight of comparisons among all neural networks is included to provide a global view on computational intelligence with neural networks in medical imaging.
\end{abstract}

Indexing terms: neural networks, medical imaging analysis, and intelligent computing.

\section{Introduction}

Inspired by the way how biological nervous systems such as human brains process information, an artificial neural network (ANN) is an information processing system which contains a large number of highly interconnected processing neurons. These neurons work together in a distributed manner to learn from the input information, to coordinate internal processing, and to optimise its final output. For the past decades, neural networks have been successfully applied to a wide range of areas, including computer science, engineering, theoretical modelling, and information systems, and medical imaging is another fruitful area for neural networks to play crucial roles in resolving problems and providing solutions. As numerous algorithms have been reported in the literature applying neural networks to medical image analysis, we provide a focused survey on computational intelligence with neural networks in terms of: (i) computer-aided detection and diagnosis with specific coverage on mammogram analysis towards breast cancer screening; (ii) segmentation and edge detection for medical image content analysis; (iii) medical image registration and (iv) other applications covering medical image compression etc, providing a global view on the variety of neural network applications and their potential for further research and developments. 
Neural network applications in computer-aided diagnosis represent the main stream of computational intelligence in medical imaging [1 14]. Their penetration and involvement are almost comprehensive for all medical problems due to the fact that: (i) neural networks have the nature of adaptive learning from input information and, using a suitable learning algorithm, can improve themselves in accordance with the variety and the change of input content; (ii) neural networks have the capability of optimising the relationship between the inputs and outputs via distributed computing, training, and processing, leading to reliable solutions desired by specifications; (iii) medical diagnosis relies on visual inspection, and medical imaging provides the most important tool for facilitating such inspection and visualization.

Medical image segmentation and edge detection remains a common problem and foundational for all medical imaging applications [15 25]. Any content analysis and regional inspection requires segmentation of featured areas, which can be implemented via edge detection and other techniques. Conventional approaches are typified by a range of well researched algorithms, including watershed [15], snake modelling [16], regiongrowing [17, N26], and contour detection etc [52]. In comparison, neural network approaches exploit the learning capability and training mechanism to classify medical images into content consistent regions to complete segmentations as well as edge detections [23-25].

Another fundamental technique for medical imaging is registration, which plays important roles in many areas of medical applications [26 32]. Typical examples include wound care, disease prediction, health care surveillance and monitoring etc. Neural networks can be designed to provide alternative solutions via competitive learning, self-organising and clustering to process input features and find the best possible alignment between different images or data sets.

Other applications of ANN include data compression [33-38, 41], image enhancement and noise suppression [39, 40, 43, 44], and disease prediction [N12, N16] etc. More recently, application of ANN for functional magnetic resonance imaging (MRI) simulation becomes a new research hotspot, where certain structured ANNs are employed to simulate the functional connectivity of brain networks [N1, N6]. Due to the similar nature of ANN and human neurons, ANN has been proved to be a very useful for this new task [N23, N34].

To provide useful insights for neural network applications in medical imaging and computational intelligence, we structure the rest of this paper in six further sections, where Section 2 provides some basics about neural networks to enable beginners to understand the structure, the connections, and the neuron functionalities. After this section, four sections are organised to provide detailed descriptions of neural network applications in the areas of computer aided diagnosis, image segmentation and edge detection, image registration, and other applications. Finally, conclusion and discussions are covered in section 7, providing concluding remarks to complete the paper.

\section{Neural Networks Fundamentals}


To enable understanding of neural network, to facilitate possible repetition of those neural networks introduced and successfully applied in medical imaging, and to inspire further development of neural networks, we cover essential basics in this section to pave the way for the rest of the chapter in surveying neural networks. We start from a theoretical model of a single neuron and then introduce a range of different types of neural networks to reveal their structure, training mechanism, operation, and functions.

\subsection{Basic Structure}

The basic structure of a neuron can be theoretically modelled as shown in Figure 1 , where $X\left\{x_{\mathrm{i}}, i=1,2, \ldots, n\right\}$ represent the inputs to the neuron and $Y$ represents the output. Each input is multiplied by its weight $w_{\mathrm{i}}$, a bias $b$ is associated with each neuron and their sum goes through a transfer function $f$. As a result, the relationship between input and output can be described as follows.

$$
Y=f\left(\sum_{i=1}^{n} w_{i} x_{i}+b\right)
$$

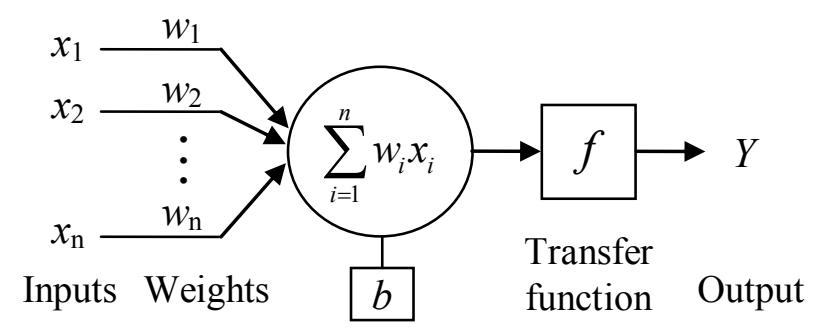

Figure 1 The model of a neuron

There are a range of transfer functions available to process the weighted and biased inputs, among which four basic transfer functions widely adopted for medical image processing are illustrated in Figure 2.

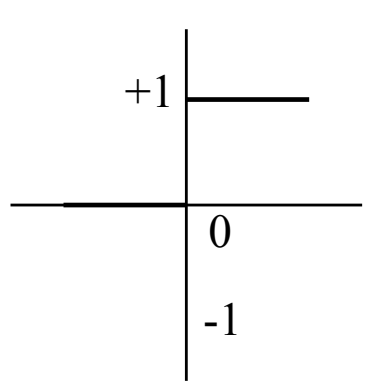

(a) HardLimit

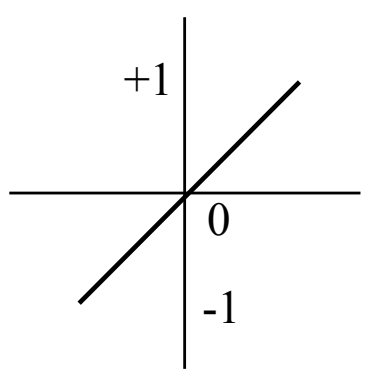

(b) Linear

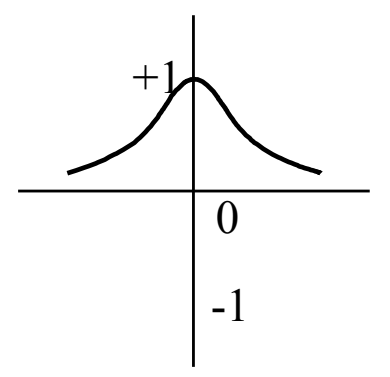

(c) RBF

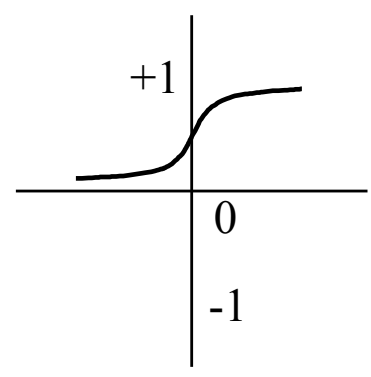

(d) Sigmoid

Figure 2 Four widely adopted transfer functions 
Via selection of suitable transfer functions and connection of neurons, various neural networks can be constructed to be trained for producing the specified outputs. Neural network types that are commonly used for medical image processing are classified as feed-forward neural networks, feedback networks and self-organizing maps. The learning paradigms for neural networks in medical image processing generally include supervised learning and unsupervised learning. In supervised learning, a network is trained using a set of inputs and outputs (targets). For each training case there will be a set of input values and one or more associated output values, and the goal is minimise the network's overall output error for all training cases by iteratively adjusting the neuron connection weights and bias values using a specific training algorithm.

In unsupervised learning, the training data set does not include any target information. Instead a function is defined that measures the suitability or accuracy of the network. This function, often referred to as a cost function, is dependent on the network's application and normally uses both the input values and the network's output value(s) to produce a cost for the current network configuration. Normally the aim of unsupervised learning is to minimise or maximise the cost for all input vectors in the training set.

\subsection{Feed-forward Network}

There are several different neural network architectures available for medical imaging applications, but one of the most common is the Feed-forward network. They often consist of multiple layers, typically including one input layer, a number of hidden layers, and an output layer. In a feed-forward network, the neurons in each layer are only connected with the neurons in the next layer. These connections are unidirectional, which means signals or information being processed can only pass through the network in a single direction, from the input layer, through the hidden layer(s) to the output layer. Fig. 3 illustrates the typical structure of a feed-forward network.

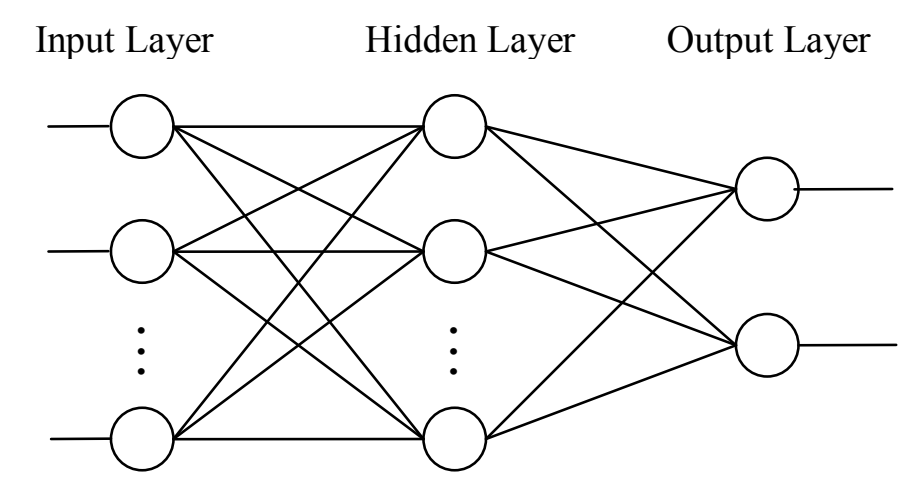

Figure 3 An example feed-forward network with a single hidden layer and two outputs

Feed-forward networks commonly use the Backpropagation (supervised) learning algorithm to dynamically alter the weight and bias values for each neuron in the network. The algorithm works by iteratively altering the connection weight values for neurons based on the error in the network's actual output value when compared to the target output value. The output error is first used to alter the weights between the final hidden 
layer and the output layer neurons by calculating the change in weight values needed to correct the output error. The weights between the final hidden layer and the previous layer (the input layer in a three-layer network) are then updated, and in this fashion the error is propagated backwards through the network to try and improve the output accuracy. The actual modification of weights is carried out using a (normally stochastic) gradient descent algorithm, where the weights are modified after each training example is present to the network. Alternatively the weights can be altered after all of the training cases have been processed in a batch fashion. A Multilayer Perceptron (MLP) is a special type of feed-forward network employing three or more layers, with nonlinear transfer functions in the hidden layer neurons. MLPs are able to associate training patterns with outputs for nonlinearly separable data. Feed-forward networks are particularly suitable for applications in medical imaging where the inputs and outputs are numerical and pairs of input/output vectors provide a clear basis for training in a supervised manner.

A Radial basis function (RBF) network is a three-layer supervised feed-forward network that uses a nonlinear transfer function (normally Gaussian) for the hidden neurons and a linear transfer function for the output neurons. The Gaussian function is applied to the net input to produce a radial function of the distance between each pattern vector and each hidden unit weight vector.

\subsection{Feed-back Network}

The feed-back (or recurrent) neural network can have signals travelling in both directions by introducing loops, propagating values from the hidden and output layers backwards to earlier layers. Their state is changing continuously until they reach an equilibrium point. They remain at the equilibrium point until the input changes and a new equilibrium needs to be found. They are potentially powerful processing tools but can become extremely complicated.

A Hopfield network is a specific type of feedback network and its inspiration is to act as a form of associative memory, in a similar way to certain parts of the human brain. The purpose of associative memory is to converge to a state remembered from training when only part of the state is presented as an input. The Hopfield network has no special input or output neurons; all neurons are both input and output, and all are connected to every other neuron in both directions. After receiving the input simultaneously by all the neurons, they output to each other and the process does not stop until a stable state is reached. In a Hopfield network, it is simple to set up the weights between neurons in order to attempt to set up a desired set of patterns as stable class patterns. They are potentially useful for medical imaging applications such as tumour classification where the output value (e.g. benign or malignant) must be derived from partial or similar patterns to those seen during training.

\subsection{Self-Organising Map}

Quite different from the above networks types, a Kohonen Neural Network (Self-Organising Map, SOM) learns to map input values to an (often two-dimensional) output space. SOMs maintain the topology of the input data 
while reducing the dimensionality, making them particularly useful for visualisation problems. SOMs can also be especially useful for medical imaging applications such as edge detection and segmentation, as their ability to automatically organise their neuron structures based on the topographical structure of the inputs can serve either as a first step in an algorithm incorporating many different approaches, or as a stand-alone method of dimensionality reduction and pattern recognition. Each neuron in a SOM stores a weight vector (an array of weights), each of which corresponds to one of the inputs in the data and a representation of its position within the map. When presented with a new input pattern, the neuron whose weight is closest in Euclidian space to the new input pattern is allowed to adjust its weights so that it gets closer to the input pattern. This type of training process is known as competitive learning, and is of course an extension of unsupervised learning.

In Kohonen neural networks, each neuron is fed by input vector (data point) $\mathrm{x} \in R^{\mathrm{n}}$ through a weight vector $\mathrm{w} \in R^{\mathrm{n}}$. Each time a data point is input to the network, only the neuron $j$ whose weight vector resembles most the input vector, is selected to fire, according to the following rule:

$$
j=\arg \min \left(\|x-w\|^{2}\right), \quad i=1,2, . . m
$$

The firing or winning neuron $j$ and its neighbouring neurons $i$ have their weight vectors $w$ modified according to the following rule:

$$
w_{i}(t+1)=w_{i}(t)+h_{i j}\left(\left\|r_{i}-r_{j}\right\|, t\right) \cdot\left(x(t)-w_{i}(t)\right),
$$

Where $h_{i j}\left(\left\|r_{i}-r_{j}\right\|, t\right)$ is a kernel defined on the neural network space as a function of the distance $\left\|r_{i}-r_{j}\right\|$ between the firing neuron $j$ and its neighbouring neurons $i$, and the time $t$ here defines the number of iterations. Its neighbouring neurons modify their weight vectors so they also resemble the input signal, but less strongly, depending on their distance from the winner.

\subsection{Training of Neural Network}

The training of a neural network can be a computationally expensive process, particularly where a combination of large network size, sometimes necessary to provide sufficient discriminatory power, and a large training dataset with many features and training examples results in a very high number of iterations of the training algorithm. Increasingly powerful hardware continues to alleviate this problem but it should be taken into account when considering the use of ANNs. In addition, how to deal with imbalanced datasets in training the ANN is also an important issue for the overall performance [N3].

Neural networks trained using supervised learning are susceptible to so-called overfitting, whereby they learn to correctly model the input-output relationship for the training examples to a high degree of accuracy, but 
perform extremely poorly on testing data that was not used during the training process. Overfitting can occur when the training data is not of sufficient size, when it is not representative of the entire feature space, or most significantly when the dimensionality of the data is too high.

This inability to generalise for unseen data renders a trained network useless for subsequent use in real applications, and several approaches are used to try and prevent overfitting from occurring. Gathering data to form the largest dataset possible, and maximising the distribution of training examples throughout the feature space can minimise the chances of overfitting. An extremely important pre-processing step before training a neural network is to select only the most relevant features from the dataset, reducing the amount of potentially irrelevant features that the network could overfit to, and often as a highly related side-effect increasing the accuracy of the network outputs.

Additionally, networks are often trained only until one or more criteria are met. Often this will involve training the network for a specified number of iterations and then stopping, or using an external validation set, not presented as training examples, to measure the output accuracy during training and stopping if and when the accuracy on validation data begins to fall while accuracy on training data rises.

From the next section onwards, detailed descriptions are provided for computational intelligence in medical imaging with neural networks, and their applications over the recent years are classified into four categories: computer aid diagnosis, image segmentation, registration and other applications. Each section gives more details on an application in one of these categories and overviews the other relevant applications. The comparison between neural networks is made in the concluding section.

\section{Neural Networks for Computer Aided Detection and Diagnosis}

Neural networks have been incorporated into many computer-aided diagnosis systems, most of which distinguish

the cancerous signs from normal tissues. Generally, these systems enhance the images first and then extract interesting regions from the images. The values of many features are calculated based on the extracted regions and are forwarded to neural works that make decisions in terms of learning, training and optimizations. Among all applications, early diagnosis of breast cancers and lung cancers represents the most typical examples in the developed computer aided detection or diagnosis (CAD) systems. Some relevant survey papers can be found in [N4, N9, N28].

\subsection{Detection and Diagnosis of Breast Cancer using Digital Mammograms}

Ge et al. [1] developed a computer-aided detection system to identify microcalcification clusters automatically on full field digital mammograms (FFDMs). The whole system includes six stages: pre-processing; image 
enhancement; segmentation of microcalcification candidates; false positive (FP) reduction for individual microcalcifications; regional clustering; and FP reduction for clustered microcalcifications.

To reduce FP individual microcalcifications, a convolution neural network $(\mathrm{CNN})$ was employed to analysis $16 \times 16$ region of interest centred at the candidate derived from segmentations. CNN was designed to simulate the vision of vertebrate animals and can be considered as a simplified vision machine designed to perform the classification of the regions into two output types: disease and non-disease. Their CNN contains an input layer with 14 neurons, two hidden layers with 10 neurons each, and one output layer. The convolution kernel sizes of the first group of filters between the input and the first hidden layer were designed as $5 \times 5$, and those of the second group of filters between the first and second hidden layers were $7 \times 7$. The images in each layer were convolved with convolution kernels to obtain the pixel values to be transferred to the following layer.

The logistic sigmoid function was chosen as the transfer function for both the hidden neurons and output neurons. An illustration of the neural network structure and its internal connections between the input layer, hidden layer and output layers is given in Figure 4.

The convolution kernels are organized in a way to emphasize a number of image characteristics rather than those less correlated values obtained from feature spaces for input. These characteristics include: (a) the horizontal versus vertical information; (b) local versus non-local information and (c) image processing (filtering) versus signal propagation[2].

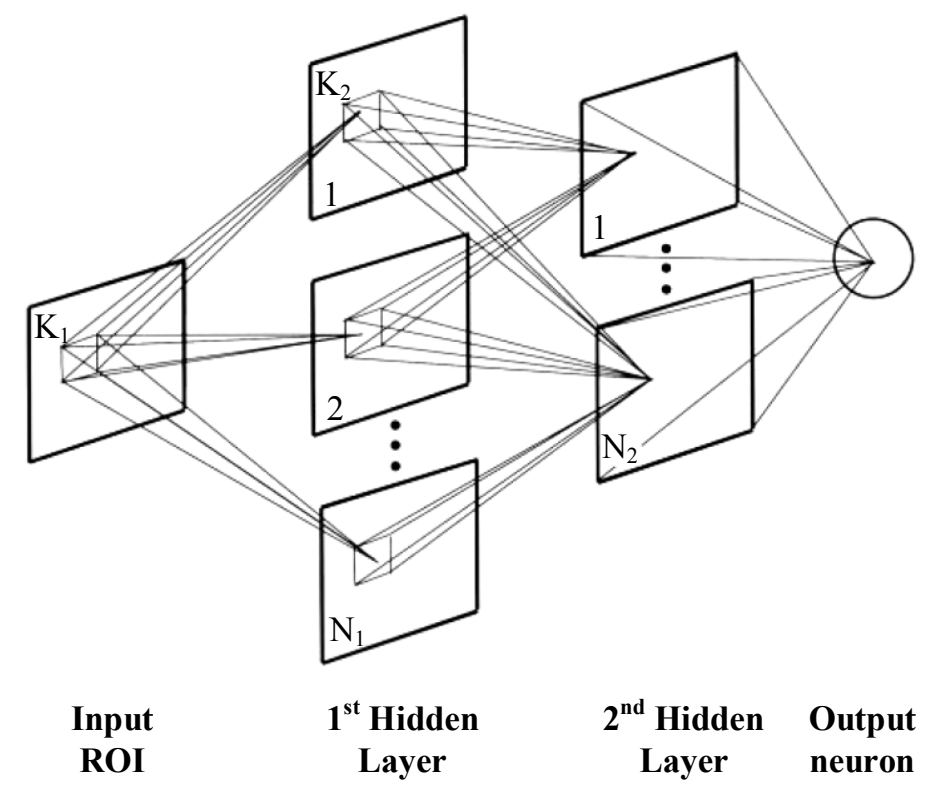

Figure 4 Schematic diagram of a $C N N$ 
The CNN was trained using back-propagation learning rule with the sum-of-squares error (SSE) function, which allows a probabilistic interpretation of the CNN output, i.e. the probability of correctly classifying the input sample as a true microcalcification ROI.

At the stage of FP reduction for clustered microcalcifications, morphological features (such as the size, the mean density, the eccentricity, the moment ratio, the axis ratio features and number of microcalcifications in a cluster) and features derived from the $\mathrm{CNN}$ outputs (such as the minimum, the maximum and the mean of the CNN output values) were extracted from each cluster. A total of 25 features (21 morphological features plus 4 $\mathrm{CNN}$ features) were extracted for each cluster. A linear discriminating analysis classifier was then used to differentiate clustered microcalcifications from false positives. The Stepwise LDA feature selection involves the selection of three parameters.

In their study, a set of 96 images is split into a training set and a validation set, each with 48 images. An appropriate set of parameters is selected by searching in the parameter space for the combination of three parameters of LDA that could achieve the highest classification accuracy with a relatively small number of features in the validation set. Then the three parameters of LDA are used to select a final set of features and LDA coefficients by using the entire set of 96 training images which contain 96 TP and over 500 FP clusters. The trained classifier is applied to a test subset to reduce the false positives (FP) in the CAD system, and through ROC analysis was shown to achieve FP reduction rates of $86 \%, 74 \%$ and $72 \%$ at sensitivities of $70 \%, 80 \%$ and $90 \%$ respectively when compared to classification without the CNN/LDA approach.

To develop a computerized scheme for the detection of clustered microcalcifications in mammograms, Nagel et al. [3] examined three methods of feature analysis: rule based (the method currently used), an artificial neural network (ANN), and a combined method. The ANN method uses a three layer error-back-propagation network with five input units corresponding to the radiographic features of each microcalcification, and one output unit corresponding to the likelihood of being a microcalcification. The reported work reveals that two hidden units are insufficient for good performance of the ANN and it is necessary to have at least three hidden units to achieve adequate performances. However, the performance is not improved any further when the number of hidden units is increased over three. Therefore, the finalised ANN has five inputs, three hidden units, and one output unit. The hybrid approach incorporating both a rule-based classifier and an ANN achieved an error rate of 0.8 false positives per image at $83 \%$ sensitivity, compared to 1.9 and 1.6 for the rule-based method and the ANN alone respectively.

Papadopoulossa et al. [ 4 ] presented a hybrid intelligent system for the identification of microcalcification clusters in digital mammograms, which can be summarised in three-steps: (a) preprocessing and segmentation, (b) regions of interest (ROI) specification and (c) feature extraction and classification. In the classification schema, 22 features are automatically computed which refer either to individual microcalcifications or to groups of them. The reduction of false positive cases is performed using an intelligent system containing two subsystems: a rule-based and a neural network based. The rule construction procedure 
consists of the feature identification step as well as the selection of the particular threshold value for each feature. Before using the neural network, the reduction in the number of features is achieved through principal component analysis (PCA), which transforms each 22-dimensional feature vector into a 9-dimensional feature vector as the input to the neural network. The neural network that is used for ROI characterisation is a feedforward neural network with sigmoid hidden neuron (Multiplayer Perceptron-MLP).

In Halkiotis [N18], ANN along with mathematical morphology is employed for the detection of clustered microcalcifications even under a non-uniform background. Considering each mammogram as a topographic representation, each microcalcification appears as an elevation constituting a regional maximum. Morphological filters are applied to suppress noise and regional maxima that do not correspond to calcifications. Two multi-layer perceptrons (MLP) and two radial basis function neural networks (RBFNN) with different number of hidden nodes are applied for classification. The MLP with ten hidden nodes achieved the best classification score with a true positive detection rate of $94.7 \%$ and 0.27 false positives per image.

Verma et al [N17] proposed a soft cluster neural network (SCNN) for the classification of suspicious areas in digital mammograms. The idea of soft clusters was employed to increase the generalisation ability of ANN by providing a mechanism to more aptly depict the relationship between the input features and the subsequent classification as either a benign or malignant class. Soft clusters with least square based optimisation made the training process faster and avoid iterative processes. The proposed neural network technique was tested on the DDSM benchmark database, and the accuracy achieved was over $93 \%$ in comparison with $83 \%$ from kmeans clustering.

Christoyiani et al. [5] presented a method for fast detection of circumscribed mass in mammograms employing a RBF neural network (RBFNN). In the method, each neuron output is a nonlinear transformation of a distance measure of the neuron weights and its input vector. The non-linear operator of the RBFNN hidden layer is implemented using a Cauchy-like probability density function. The implementation of RBFNN could be achieved by using supervised or unsupervised learning algorithms for an accurate estimation of the hidden layer weights. The K-means unsupervised algorithm is used to estimate the hidden-layer weights from a set of training data containing statistical features from both circumscribed lesions and normal tissue. After the initial training and the estimation of the hidden-layer weights, the weights in the output layer are computed by using Wincerfilter theory, or minimizing the mean square error (MSE) between the actual and the desired filter output. The method was tested using the The MIAS MiniMammiographic Database, and achieved a mean overlap value of 0.868 for true positives for both normal and abnormal mammograms.

Patrocinio et al. [6] demonstrate that only certain features such as irregularity, number of microcalcifications in a cluster, and cluster area, are needed as the inputs of a neural network to separate images into two distinct classes: suspicious and probably benign. Setiono [7] developed an algorithm by pruning a feedforward neural network, which produces high accuracy rates for breast cancer diagnosis with small number of connections. The algorithm extracts rules from a pruned network by considering only a finite number of hidden 
unit activation values. Connections in the network are allowed only between input units and hidden units as well as between hidden units and output units. The algorithm finds and eliminates as many unnecessary network connections as possible during the training process. The accuracy of the extracted rules from the pruned network is almost as high as the accuracy of the original network.

The abovementioned applications cover different aspects of applying neural networks such as the number of neurons in the hidden layer, the reduction of features in classifications, the reduction of connections for better efficiency. Similar improvements could be made in applying ANN to other practical utilisations rather than just in identifying microcalcification clusters. For other approaches rather than ANN in detection and classification of microcalcifications and masses in mammograms, please refer to the papers [N28] and [N29].

\subsection{Detection and Diagnosis of Lung Diseases}

ANNs also plays an important role in detecting cancerous signs in lungs. $\mathrm{Xu}$ et al. [8] developed an improved computer-aided diagnosis (CAD) scheme for the automated detection of lung nodules in digital chest images to assist radiologists, who could miss up to $30 \%$ of the actually positive cases in their daily practice. In the CAD scheme, nodule candidates were selected initially by multiple gray-level thresholds of the difference image (subtraction of a signal-enhanced image and a signal suppressed image) and then classified into six groups. Between $50 \%$ and $70 \%$ of false positives were eliminated by adaptive rule-based tests and an ANN.

Zhou et al. [9] proposed an automatic pathological diagnosis procedure named Neural Ensemble-based Detection (NED) that utilizes an ANN ensemble to identify lung cancer cells in the specimen images of needle biopsies obtained from the bodies of the subjects to be diagnosed. An ANN ensemble is a learning paradigm where several ANNs are jointly used to solve a problem. The ensemble is built on a two-level ensemble architecture and the predictions of those individual networks are combined by plurality voting.

Keserci et al. [10] developed a computer-aided diagnosis scheme for automated detection of lung nodules in digital chest radiographs based on a combination of morphological features and the wavelet snake. In their scheme, an ANN was used to efficiently reduce false positives by using the combined features. The scheme was applied to a publicly available database of digital chest images for pulmonary nodules. Qian et al. [11] trained a computer-aided cytologic diagnosis (CACD) system to recognize expression of the cancer biomarkers histone H2AX in lung cancer cells and then tested the accuracy of this system to distinguish resected lung cancer from preneoplastic and normal tissues. The major characteristics of CACD algorithms are to adapt detection parameters according to cellular image contents. Coppini et al. [12] described a neural-network-based system for the computer aided detection of lung nodules in chest radiograms. The approach is based on multi-scale processing and feed-forward neural networks that allow an efficient use of a priori knowledge about the shape of nodules and the background structure.

\subsection{Detection and Diagnosis in MRI}


ANN has also been widely applied in diagnosis of diseases in MR images. In Guo et al [N8], a computer-aided diagnostic system was proposed to classify rat liver lesions from MR imaging. Six parameters of texture characteristics and Variance of 161 ROIs were calculated and assessed by gray-level co-occurrence matrices, then fed into a BP neural network (NN) classifier to classify the liver tissue into two classes namely cirrhosis and HCC. The accuracy of classification of HCC nodules from cirrhosis achieved was 91.67\%.

In Yamashita et al [N10], ANN was utilised to evaluate the performance of radiologists for differential diagnosis of intra-axial cerebral tumors on MR Images. A single 3-layer feed-forward ANN with a LevenbergMarquardt algorithm was employed to differentiate among 4 categories of tumors with use of 2 clinical parameters and 13 radiologic findings in MR images. Subjective ratings for the 13 radiologic findings were provided independently by 2 attending radiologists. In total 126 cases were used for training and testing of the ANN based on a leave-one-out-by-case method. In the observer test, MR images were viewed by 9 radiologists, first without and then with ANN outputs. The averaged area under the ROC curve for ANN alone was 0.949. The diagnostic performance of the 9 radiologists increased from 0.899 to 0.946 when they used ANN outputs.

Döhler et al [N11] proposed a cellular ANN for the detection of hippocampal sclerosis in MRI. Using an exemplary database that consisted of a large number of volumes of interest extracted from T1-weighted magnetic resonance images from 144 subjects, the authors demonstrated that the network allowed classifying brain tissue with respect to the presence or absence of mesial temporal sclerosis. Results indicated the general feasibility of the proposed computer-aided systems for diagnosis and classification of images generated by medical imaging systems.

\subsection{Detection and Diagnosis of Other Diseases}

Apart from the applications in breast cancer and lung cancer, ANN has been adopted in many other analyses and diagnosis. Mohamed et al. [13] compare bone mineral density (BMD) values for healthy persons and identify those with conditions known to be associated with BMD obtained from Dual X-ray absorptiometry (DXA). An ANN was used to quantitatively estimate site-specific BMD values in comparison with reference values obtained by DXA (i.e. BMDspine, BMDpelvis, and BMDtotal). Anthropometric measurements (i.e. sex, age, weight, height, body mass index, waist-to-hip ratio, and the sum of four skinfold thicknesses) were fed to an ANN as independent input variables. The estimates based on four input variables were generated as output and were generally identical to the reference values for all studied groups.

Scott [14] tried determining whether a computer based scan analysis could assist clinical interpretation in this diagnostically difficult population. Artificial neural networks (ANNs) were created using only objective image-derived inputs to diagnose the presence of pulmonary embolism. The ANN predictions performed comparably to clinical scan interpretations and with the results of angiography.

In Zhang et al [N2], a computer-aided diagnosis system named LiverANN was proposed for classifying the pathologies of focal liver lesions into five categories using the artificial neural network (ANN) technique. On 
each MR image, a region of interest (ROI) in the focal liver lesion was delineated by a radiologist. The intensity and homogeneity within the ROI were calculated automatically, producing numerical data that were analyzed by feeding them into the LiverANN as inputs. Of the 320 MR images obtained from 80 patients with liver lesions, the ANN classifier can achieve a training accuracy of $100 \%$ and a testing accuracy of $93 \%$ in classifying the cases into five classes.

Tägil et al [N14] employed ANN for quality assurance of image reporting in terms of automatic interpretation in myocardial perfusion imaging. The networks were used to identify potentially suboptimal or erroneous interpretations of myocardial perfusion scintigrams (MPS). Reversible perfusion defects in each of 5 myocardial regions, as interpreted by one experienced nuclear medicine physician, were assessed by ANN in 316 consecutive patients undergoing MPS. After training, the ANNs were used to select 20 cases in each region that were more likely to have a false clinical interpretation. These cases, together with 20 detected control cases with no likelihood of false clinical interpretation, were randomly presented to three experienced physicians for a consensus re-interpretation. Due to small and mild perfusion defects and localization of defects, clinical routine interpretation by an experienced nuclear medicine expert and ANN differed in 53 of the 200 cases. The results demonstrated that ANN could identify those MPS that might have suboptimal image interpretations.

In Pan et al [N22], BP based ANN was utilised for bleeding detection in wireless capsule endoscopy (WCE). Colour texture features distinguishing the bleeding regions from non-bleeding regions were extracted in RGB and HSI colour spaces, and used as the feature vector inputs to the ANN to recognize the bleeding regions. The experiments demonstrated that the bleeding regions could be correctly recognized with a sensitivity of $93 \%$ and a specificity of $96 \%$.

\subsection{Summary}

In all the applications mentioned above, the roles of ANNs have a common principle in the sense that they all are applied to reduce FP detections in both mammograms and chest images via examining the features extracted from the suspicious regions. As a matter of fact, ANNs are not limited to academic research, but also play important roles in commercially available diagnosis systems, such as ImageChecker for mammograms.

\section{Others}

According to the results reported in [N29] and [n16], it is interesting to note that combined classifiers tend to yield better results than single ones. However, ANN still can generate good results as an expert mammographer [N29], although in some work it is suggested that SVM may produce better results in detecting microcalcification [N27]. In Chen et al [N7], it is found that the diagnostic performance of ANN is not different from that of SVM and LRA (Logistic regression analysis) as demonstrated by ROC curve analysis. The inconsistency here may refer to the differences between the test data and test conditions, i.e. how much of the data is used for training and how the classifiers are optimised et al. 


\section{Neural Networks for Medical Image Segmentation and Edge Detection}

Medical image segmentation is a process for dividing a given image into meaningful regions with homogeneous properties. Image segmentation is an indispensable process in outlining boundaries of organs and tumours, and in the visualization of human tissues during clinical analysis. Therefore, segmentation of medical images is very important for clinical research, diagnosis, and applications, leading to requirement of robust, reliable and adaptive segmentation techniques.

\subsection{Segmentation}

Kobashi et al. [15] proposed an automated method to segment the blood vessels from 3D time of flight (TOF) MRA volume data. The method consists of three steps: (1) removal of the background, (2) volume quantization, and (3) classification of primitives by using an artificial neural network.

After volume quantization by using a watershed segmentation algorithm, the primitives in the MRA image stand out. To further improve the result of segmentation, the obtained primitives have to been separated into the blood vessel class and the fat class. Three features and a feed-forward three-layered neural network are adopted for the classification. Compared with the fat, the blood vessel is like a tube - long and narrow. To this end, two features including vascularity and narrowness were introduced to measure such properties. As the histogram of blood vessels is quite different from that of the fat in shapes, a third feature, histogram consistency, is added for further improvement of the segmentation.

The feed-forward NN is composed of 3 layers: an input layer, a hidden layer and an output layer. The structure of the described neural network is illustrated in Figure 5.

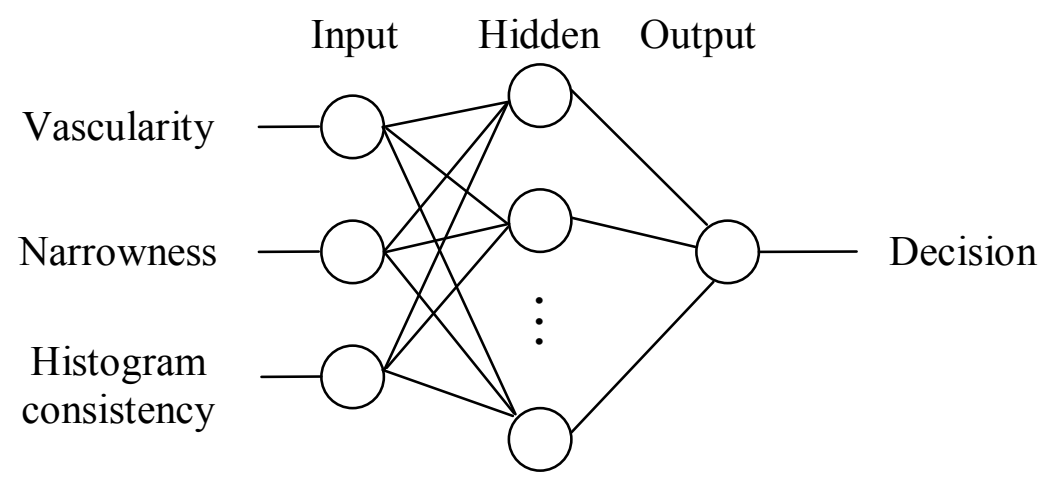

Figure 5 Three layer feed-forward neural network

As seen, three input units are included at the input layer, which is decided by the number of features extracted from medical images. The number of neuron in the output layer is one to produce and represent two classes. The number of neurons in the hidden layer is usually decided by experiments. Generally, a range of different numbers is tried in the hidden layer, and the number that achieves the best training results is selected. 
In the proposed method, the ANN classifies each primitive, which is a clump of voxels, by evaluating the intensity and the 3D shape.

In their experiments, the ANN was trained using 60 teaching data sets derived from an MRA data set. Each primitive is classified into the blood vessel (indicated by the value of 1 ) or the fat (indicated by the value of 0 ) and the values of the three features are calculated. All these values were fed into the feed-forward ANN for training the weights of the neurons. Seven new MRA data, whose primitives were unclassified, were fed into the trained NN for testing. The segmentation performance is measured by the value of accuracy as defined below, and the rate achieved by the reported algorithm is $80.8 \%$.

$$
\text { Accuracy }=\frac{\text { Number of correctly classified primitives }}{\text { Total number of primitives }} \times 100 \%
$$

Apart from the work proposed by Kobashi in ANN based segmentation, there are many applications for the images generated by CT and MRI. Middleton et al. [16] combined use of a MLP and active contour model ('snake') to segment structures in magnetic resonance (MR) images. The reported work can be highlighted by the following two steps:

1) The perceptron is trained to produce a binary classification of each pixel as either a boundary or a nonboundary;

2) Subsequently, the resulting binary (edge-point) image forms the external energy function for a snake model, which is used to link the candidate boundary points into a continuous and closed contour.

Lin [17] applied a Hopfield neural network with penalized fuzzy c-means technique (called PFHNN) to medical image segmentation. In the algorithm, the pixels with their first and second order moments constructed from their $n$ nearest neighbours as a training vector are mapped to a two-dimensional Hopfield neural network for the purpose of classifying the image into suitable regions.

Lin et al. [18] generalized Kohonen's competitive learning (KCL) algorithm with fuzzy and fuzzy-soft types called fuzzy KCL (FKCL) and fuzzy-soft KCL (FSKCL). These KCL algorithms fuse the competitive learning with soft competition and fuzzy c-means (FCM) membership functions. These generalized KCLs were applied to MRI and MRA ophthalmological segmentations. It is found that these KCL-based MRI segmentation techniques are useful in reducing medical image noise effects using a learning mechanism. The FSKCL algorithm is recommended for use in MR image segmentation as an aid to small lesion diagnosis.

Dokur [19] proposed a Quantizer Neural Network (QNN) for the segmentation of MR and CT images. QNN is a novel neural network structure, which is trained by genetic algorithms. It was comparatively examined with a multilayer perceptron and a Kohonen network for the segmentation of MR and CT head images. The QNN was reported to have the best classification performance with fewer neurons after a short training time. 
Stalidis et al. [20] presented an integrated model-based processing scheme for cardiac magnetic resonance imaging (MRI), embedded in an interactive computing environment suitable for quantitative cardiac analysis, which provides a set of functions for the extraction, modeling, and visualization of cardiac shape and deformation. In the scheme, a learning segmentation process incorporating a generating-shrinking neural network is combined with a spatiotemporal parametric modeling through functional basis decomposition.

Chang et al. [21] developed an approach for medical image segmentation using a fuzzy Hopfield neural network based on both global and local gray-level information. The membership function simulated with neuron outputs is determined using a fuzzy set, and the synaptic connection weights between the neurons are predetermined and fixed to improve the efficiency of the neural network.

Shen et al. [22] proposed a segmentation technique based on an extension to the traditional fuzzy cmeans (FCM) clustering algorithm. In the paper, a neighbourhood attraction, which is dependent on the relative location and features of neighbouring pixels, is shown to improve the segmentation performance and the degree of attraction is optimized by a neural-network model. Synthetic and real brain MR images with different noise levels are segmented to demonstrate the superiority of the proposed technique compared to other FCM-based methods.

Fu et al [N21] proposed an automatic hybrid model, in which the statistical expectation maximization (EM) and the spatial pulse coupled neural network (PCNN) were integrated for brain MRI segmentation. In addition, an adaptive mechanism was developed to fine tune the PCNN parameters. The EM model served two functions including evaluation of the PCNN image segmentation and adaptive adjustment of the PCNN parameters for optimal segmentation. They concluded the adaptive EM-PCNN yielded the best results for gray matter and brain parenchyma segmentation.

\subsection{Edge Detection}

Chang et al [23] designed a two-layer Hopfield neural network called the competitive Hopfield edge-finding neural network (CHEFNN) to detect the edges of CT and MRI images. The CHEFNN extends the one-layer 2-D Hopfield network at the original image plane to a two-layer 3-D Hopfield network with edge detection to be implemented on its third dimension. With the extended 3-D architecture, the network is capable of incorporating a pixel's contextual information into a pixel-labelling procedure. As a result, the effect of tiny details or noises will be effectively removed by the CHEFNN and the drawback of disconnected fractions can be overcome. In addition, they [24] discovered that high-level contextual information cannot be incorporated into the segmentation procedure in techniques using traditional Hopfield neural networks and thus proposed contextual constraint-based Hopfield neural cube (CCBHNC) for image segmentation. The CCBHNC uses a threedimensional architecture with pixel classification implemented on its third dimension. With the threedimensional architecture, the network is capable of taking into account each pixel's feature and its surrounding contextual information, achieving up to $95.86 \%$ segmentation accuracy on real MRI images. Recently, still for 
the edge detection, Chang [25] presented a special design Hopfield neural network called the contextual Hopfield neural network (CHNN). The CHNN maps the 2-D Hopfield network at the original image plane. With the direct mapping, the network is capable of incorporating pixels' contextual information into an edge-detecting procedure. As a result, the CHNN can effectively remove the influence of tiny details and noise.

In Suzuki et al [N25], a neural edge detector (NED) is proposed to extract contours from left ventriculograms. A modified multilayer neural network is employed and trained using a modified backpropagation algorithm through supervised learning from a set of images with manually extracted edges by a cardiologist. It is found that the NED is able to extract the contours in agreement the ground truth, where an average contour error of $6.2 \%$ and an average difference between the ejection fractions at $4.1 \%$ are reported.

\subsection{Summary}

Although most of these applications are developed based on CT or MRI images but the neural networks adopted are quite different. ANN is able to reduce the influence of noise in the image and hence make the segmentation more robust. Further, ANN could also classify different tissues and then combine them according to the requirements of segmentations, which is beyond the power of traditional segmentation.

New part!

\section{Neural Networks for Medical Image Registration}

Image registration is the process of transforming the different sets of data into one coordinate system. Registration is necessary to enable the comparison, integration and fusion of images from different measurements, which may be taken at different points in time from the same modality or obtained from the different modalities such as CT, MR, Angiography and Ultrasound. Medical imaging registration often involves elastic (or non-rigid) registration to cope with elastic deformations of the body parts imaged. Non-rigid registration of medical images can also be used to register a patient's data to an anatomical atlas. Medical image registration is pre-processing needed for many medical imaging applications with strong relevance to the result of segmentation and edge detection.

\subsection{Techniques}

Generally, image registration algorithms could be classified into two groups: area based methods and feature based methods. For area based image registration methods, the algorithm looks at the structure of the image via correlation metrics, Fourier properties and other means of structural analysis. Most feature based methods fine tunes its mapping to the correlation of image features: lines, curves, points, line intersections, boundaries, etc.

To measure the volume change of lung tumour, Matsopoulos et al. [26] proposed an automatic threedimensional non-rigid registration scheme that applied self-organizing maps (SOMs) to thoracic computed 
tomography (CT) data of patients for establishing correspondence between the feature points. The practical implementation of this scheme could provide estimations of lung tumour volumes during radiotherapy treatment planning. In the algorithm, the automatic correspondence of the interpolant points is based on the initialization of the Kohonen neural network model capable to identify 500 corresponding pairs of points approximately in the two CT sets $S_{1}$ and $S_{2}$. An overview of the described algorithm is illustrated in Figure 6.

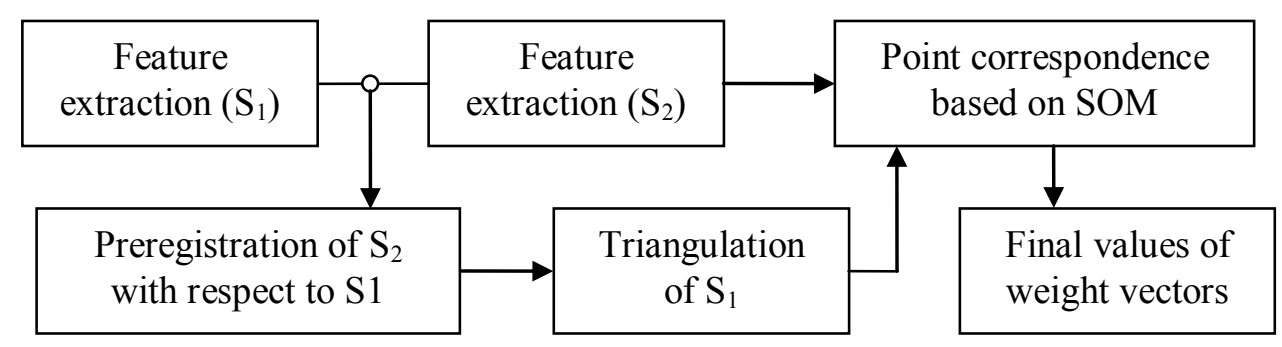

Figure 6 The elastic registration scheme

In the algorithm, two sets of points are defined: $S_{2}$ is the set of points for vertebrae, ribs and blades segmented from the reference data, and $S_{1}$ the set of points for the same anatomical structures from the second dataset called float data. Pre-registration takes place between these sets of points, and triangulation of $\mathrm{S}_{1}$ is performed. The preregistration process is applied in three dimensions and is applied in order to realign the two datasets in all coordinate. After pre-registration, two steps are performed to obtain the interpolant points, which are described below:

1. Triangulating $\mathrm{S}_{1}$ and producing a wire frame based on the topology of $\mathrm{S}_{1}$;

The triangulation is based on Feitzke's work [27] and is performed by defining a SOM with the following characteristics:

a. A grid of neurons with 20 rows by 100 columns $(20 \times 100)$ is chosen for the specific implementation.

b. The initial weighting vectors of the neurons of the grid are set equal to the coordinates of a set of points extracted from an enclosing surface, typically a cylindrical surface.

c. The input to the neural network consists of the Cartesian coordinates of the set of points that need to be triangulated.

After the process of adaptation of the neural network, the weighting vectors of the neurons have values identical to the appropriate points of $S_{1}$. A wire frame consisting of one node for each neuron can be constructed, with Cartesian coordinates of each node equal to the weight vector of the corresponding neuron. The wire frame is triangulated according to the connectivity of the neurons.

2. Establishing a SOM in terms of the topology of $\mathrm{S}_{1}$ and training the SOM by using $\mathrm{S}_{2}$; 
The search for corresponding points is based on replicating the topology of the set $S_{1}$ on the input layer of a SOM model. In the SOM model, one neuron is assigned to each node of the wired frame and the connections between the neurons are identical with the connections of the wired frame. No connection between two neurons is allowed if the two corresponding nodes are not directly connected on the float set. The initial weight vector of the neurons is the Cartesian co-ordinates of the corresponding wired frame nodes in the $3 \mathrm{D}$ space.

The training of the network is performed by presenting the network with the coordinates of randomly selected points sampled from the reference set $S_{2}$. The neuron with weight vector closest to signal is selected to fire. The firing neuron adjusts its weight vector and its neighbouring neurons modify their weight vectors as well but less strongly. The neighbouring neurons are confined to a window of $3 \times 3$ neurons throughout the network training.

The convergence of the SOM network during the triangulation of $\mathrm{S}_{1}$ set of points results in a triangulated subset of points $\left(S_{1}{ }^{\prime}\right)$. Each node of subset $S_{1}$ ' corresponds to a neuron of the SOM network $(20 \times 100$ neurons $)$, whose initial weighting vector $\left(x_{0}, y_{0}, z_{0}\right)$ of $\mathrm{S}_{1}$ is equal to the initial Cartesian coordinates of this node. In $\mathrm{S}_{1}$, this node is displaced to new coordinates and equal to the final weighting vector $\left(x_{1}, y_{1}, z_{1}\right)$. The new position always coincides with a point in $\mathrm{S}_{2}$.

Although SOM lateral interactions between neurons generate a one to one point correspondence, more than one point from S1' may correspond to one point in S2. However, most of such point mismatches are avoided by using a distance threshold criterion that excludes corresponding points exceeding a distance more than five voxels. This process also prohibits excessive deformation of the final warped image. Therefore, the total number of successful corresponding points is reduced to approximately 500 pairs of points for all patient data.

SOM has also been used in many other registration-related applications. Shang et. al. [28] developed an automatic method to register computed tomography (CT) and magnetic resonance (MR) brain images by using first principal directions of feature images. In the method, principal component analysis (PCA) neural network is used to calculate the first principal directions from feature images, and then the registration is accomplished by simply aligning feature images' first principal directions and centroids.

Coppini [29] presented a general approach to the problem of image matching which exploits a multiscale representation of local image structure. In the approach, a given pair of images to be matched, named target and stimulus respectively, are represented by Gabor Wavelets. Correspondence is computed by exploiting the learning procedure of a neural network derived from Kohonen's SOM. The SOM neurons coincide with the pixels of the target image and their weight are pointers to those of the stimulus images. The standard SOM rule is modified so as to account for image features. 
Fatemizadeh et al. [30] proposed a method for automatic landmark extraction from MR brain images. In the method, landmark extraction is accomplished by modifying growing neural gas (GNG), which is a neuralnetwork-based cluster-seeking algorithm. Using modified GNG (MGNG, a splitting-merging SOM) corresponding dominant points of contours extracted from two corresponding images are found. The contours are the boundaries of the regions generated by segmenting the MR brain image.

Di Bona et al. [31] developed the "Volume-Matcher 3D" project - an approach for a data-driven comparison and registration of three-dimensional (3D) images. The approach is based on a neural network model derived from self-organizing maps and extended in order to match a full 3D data set of a "source volume" with the 3D data set of a "target volume." In Zhang et al [N13], an automatic surface-based rigid registration system using a neural network representation was proposed. The system was applied to register $3 \mathrm{D}$ volumes of human bone structures for image-guided surgery. A multilayer perceptron neural network was used to construct a patient-specific surface model from pre-operative images. A surface representation function derived from the resultant neural network model was then employed for intra-operative registration. The optimal transformation parameters were obtained via an optimization process. Experiments using image datasets of the calcaneus and vertebrae demonstrated that the segmentation/registration system could achieve sub-voxel accuracy comparable to that of conventional techniques, and was significantly faster.

Markaki et al [N24->32] proposed automatic point correspondence of unimodal medical images using Kohonen Network. Given a pair of 2D medical images of the same anatomical region and a set of interest points in one of the images, the algorithm detected effectively the set of corresponding points in the second image, by exploiting the properties of the Kohonen self organizing maps (SOMs) and embedding them in a stochastic optimization framework. The correspondences were established by determining the parameters of local transformations of point mapping in an iterative way, using a modified competitive learning as implemented by SOMs. Experimental results from three different modalities (CT, MR and red-free retinal images) had used to validate both the accuracy and efficiency of the proposed algorithm, even in the case of noise corrupted data.

\subsection{Summary}

These applications suggest that SOM is a promising algorithm for elastic registration, which is probably due to the clustering characteristics of SOM.

Need new material

\section{Other Medical Applications using Neural Network}


In addition to the areas mentioned above, ANN has also been applied to other relevant areas such as medical image compression [33-38], enhancement [39-44], restoration [46], disease prediction [N12, N16] as well as the more recent application in functional MRI simulation [N1, N6] et al.

\subsection{Compression and Coding}

In image compression [32], medical images such as mammograms are usually quite large in size and stored in the databases inside hospitals, which brings some difficulties in image transfer over the Internet. Some researchers have applied ANN to existing compression algorithms to select interesting regions for transmission or reduce the errors during the quantization in compression [33 37, 41].

Panagiotidis et al. [33] proposed a neural network architecture to perform lossy compression of medical images. To achieve higher compression ratio while retain the significant (from medical point of view) image content, the neural architecture adaptively selects regions of interest (ROI) in the images.

Karlik [34] presented a novel and combined technique for image compression based on the Hierarchical Finite State Vector Quantization (HFSVQ) and neural networks. The algorithm performs nonlinear restoration of diffraction-limited images concurrently with quantization. The neural network is trained on image pairs consisting of a lossless compression named hierarchical vector quantization.

Meyer-Base et al. [35] developed a method based on topology-preserving neural networks to implement vector quantization for medical image compression. The method can be applied to larger image blocks and represents better probability distribution estimation methods. The quantization process is performed by a "neural-gas" network which applied to vector quantization converges quickly to low distortion errors and reaches a distortion error lower than that resulting from Kohonen's feature map or the LBG algorithm. The influence of the neural compression method on the phantom features and the mammo-graphic image is not visually perceptible up to a high compression rate.

Jaiswal et al. [36] trained a resilient back propagation neural network to encode and decode the input data so that the resulting difference between input and output images is minimized. Lo et al [37] developed a neural-network-based framework to search for an optimal wavelet kernel that can be used for a specific image processing task. In the algorithm, a linear convolution neural network was employed to seek a wavelet that minimizes errors and maximizes compression efficiency for an image or a defined image pattern such as microcalcifications in mammograms and bone in computed tomography (CT) head images.

In Dokur [N15], ANN was applied to medical images like magnetic resonance (MR), computer tomography (CT) head images and ultrasound imaging for compression and decision making, where Kohonen map and incremental self-organizing map (ISOM) were employed. In the proposed method, the image was first decomposed into blocks of $8 \times 8$ pixels, from which 2D discrete cosine transform (DCT) coefficients were computed. The dimension of the DCT coefficients vectors was reduced by low-pass filtering, a similar way like vector quantization. The decision making was realised simultaneously with compression to cluster codewords 
into several classes, which also formed a kind of segmentation of the original image. Higher compression rates with large signal to noise ratio were gained compared to the JPEG standard.

\subsection{Image Enhancement and Noise Suppression}

To enhance original images, ANN has been used to suppress unwanted signals such as noise and tissues affecting cancerous sign. Suzuki et. al. [38] proposed an analysis method that makes clear the characteristics of the trained NF (i.e. Nonlinear filters based on multilayer neural networks) and developed approximate filters that achieves very similar results but is efficient at computational cost.

To detect lung nodules overlapped with ribs or clavicles in chest radiographs, Suzuki et al. [39] developed an image-processing technique for suppressing the contrast of ribs and clavicles in chest radiographs by means of a multi-resolution massive training artificial neural network (MTANN). The structure of this neural network is illustrated in Figure 7, in which "bone" images are obtained by use of a dual-energy subtraction technique [40] as the teaching images to facilitate the neural network training. After that, the multi-resolution MTANN is able to provide "bone-image-like" images which are similar to the teaching bone images. By subtracting the bone-image-like images from the corresponding chest radiographs, they are able to produce "soft-tissue-image-like" images where ribs and clavicles are substantially suppressed.

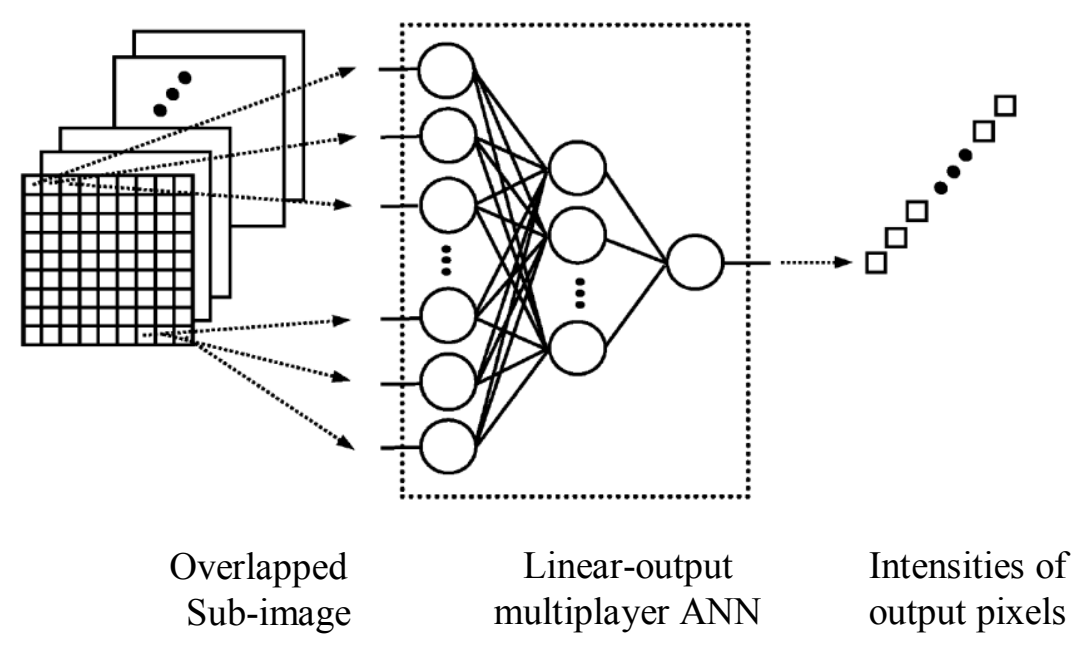

Figure 7 Architecture of MTANN

The MTANN consists of a linear-output multilayer ANN model, which is capable of operating on image data directly. The linear-output multilayer ANN model employs a linear function as the transfer function in the output layer because the characteristics of an ANN were improved significantly with a linear function when applied to the continuous mapping of values in image processing [41]. The inputs of the MTANN are the pixel values in a size-fixed sub-image and can be rewritten as $I_{x, y}=\left\{I_{1}, I_{2}, \ldots, I_{N}\right\}$, where $N$ is the number of inputs i.e. the number of pixels inside a sub-image. The output of the $n$th neuron in the hidden layer is represented by 


$$
O_{n}=f_{h}\left\{\sum_{m=1}^{N} w_{m n} \cdot I_{m}-b_{n}\right\}
$$

Where $w_{m n}$ is a weight between the $m$ th unit in the input layer and the $n$th neuron in the hidden layer, and $f_{h}$ is a sigmoid function. The output of the neuron in the output layer is represented by:

$$
f(x, y)=f_{o}\left\{\sum_{m=1}^{N_{h}} w_{m}^{O} \cdot O_{m}^{H}-b_{o}\right\}
$$

where $w_{m}^{o}$ is a weight between the $m$ th neuron in the hidden layer and the neuron in the output layer, $b_{o}$ is an offset of the neuron in the output layer.

To train MTANN, a dual-energy subtraction technique is used to obtain the teaching image $T$ (i.e. "bone" images) for suppression of ribs in chest radiographs. Input chest radiographs are divided pixel by pixel into a large number of overlapping sub-images. Each sub-image $I(x, y)$ corresponds to a pixel $T(x, y)$ in teaching image, and the MTANN is trained with massive sub-image pairs as defined below:

$$
\left\{I(x, y), T(x, y) \mid x, y \in R_{T}\right\}=\left\{\left(I_{1}, T_{1}\right),\left(I_{2}, T_{2}\right), \ldots,\left(I_{N_{T}}, T_{N_{T}}\right)\right\}
$$

where $R_{T}$ is a training region corresponding to the collection of the centres of sub-images, $N_{T}$ is the number of pixels in $R_{T}$. After training, the MTANN is expected to produce images similar to the teaching images, i.e. "bone-image-like" images. The technique was evaluated using a set of 118 images by applying the algorithm to each image and then quantitatively comparing it to a dual-energy soft-tissue image where the bone regions had been deemphasised.

Since Ribs in chest radiographs include various spatial-frequency components and it is difficult in practice to train the MTANN with a large sub-image, multi-resolution decomposition/ composition techniques are employed in the algorithm. Three MTANNs for different resolutions are trained independently with the corresponding resolution images: a low-resolution MTANN is in charge of low-frequency components of ribs, a medium-resolution MTANN is for medium-frequency components, and a high-resolution MTANN for highfrequency components. After training, the MTANNs produce a complete high-resolution image based on the images with different resolution.

Hainc et al. [42] found the artificial neural network can also be used as a kind of a sophisticated nonlinear filter on local pixel neighbourhood $(3 \times 3)$ since linear systems are not good in their sensitivity to impulse (isolated) noise.

Chen et al. [43] introduced an ANN architecture for reducing the acoustic noise level in magnetic resonance (MR) imaging processes. The proposed ANN consists of two cascaded time-delay ANN. The ANN is used as the predictor of a feedback active noise control (ANC) system for reducing acoustic noises. Preliminary 
results also show that, with the proposed ANC system installed, acoustic MR noises are greatly attenuated while verbal communication during MRI sessions is not affected.

\subsection{Functional MRI (fMRI) Simulation}

Since the mid of 1990s, functional connectivity study using fMRI has drawn increasing attention of neuroscientists and computer scientists, which opens a new window to explore functional network of human brain [N34]. Among quite a few work reported, ANN has been found as a natural way and powerful tool for simulating the connectivity and function of special areas of brain [N1, N6, N33]. A comprehensive survey in this topic can be referred to [N23], [N31] and [N34].

In Kim and Horwitz [N1], different kinds of fMRI functional connectivity are analysed to reflect the underlying interregional neural interactions, where a biologically realistic neural model is employed to simulate both neuronal activities and multiregional fMRI data from a blocked design. Topics involved include psychophysiological interaction (PPI) analysis and interregional correlation analysis, and a large-scale neural model is applied to simulate the neurobiological underpinnings of PPI. The experimental results have clearly shown that neural modelling can be used to help validate the inferences one can make about functional connectivity based on fMRI data.

In Marrellec et al [N6], a novel approach based on the partial correlation matrix is proposed to develop data-driven measures of effective connectivity in functional MRI. To achieve this target, a large-scale, neurobiologically realistic neural network model is employed to generate simulated data with both structural equation modelling (SEM) and the partial correlation approach. Unlike real experimental data, where the interregional anatomical links are not necessarily known, the links between the nodes of the neural model are fully specified for easily judging the results of SEM and partial correlation analyses. The results reported have fully validated the partial correlation method with respect to the underlying neuroarchitecture

In Guenther et al [N33], a neural model of speech acquisition and production is described that accounts for a wide range of acoustic, kinematic, and neuroimaging data concerning the control of speech movements. The components of the ANN model correspond to regions of the cerebral cortex and cerebellum, including premotor, motor, auditory, and somatosensory cortical areas. Computer simulations of the model verify its ability to account for compensation to lip and jaw perturbations

during speech. Specific anatomical locations of the model's components are estimated, and these estimates are used to simulate fMRI experiments of simple syllable production.

\subsection{Disease Prediction}

The application of ANN in prediction of outcomes using clinical data is being increasingly used. In Chiu [N12] et al, ANN model is employed for predicting skeletal metastasis in patients with prostate cancer. Through analysis of data consecutively collected from patients in five years, the predictors in terms of the patient's age 
and radioimmunometric serum PSA concentration are analysed. To assess the classification performance for clinical study, the discrimination and calibration of an ANN model is estimated and the one of the best performance is determined as four-layered perceptrons. Evaluations using the area under the receiver-operating characteristics curve and the Hosmer-Lemeshow statistics suggest that ANN appears to be a promising method in forecasting of the skeletal metastasis in patients with prostate cancer.

In Goodband et al [N16], application of ANN in image-guided radiation therapy is presented, aiming to improve the accuracy of treatment delivery by tracking tumor position and compensating for observed movement. Due to system latency it is sometimes necessary to predict tumor trajectory evolution in order to facilitate changes in beam delivery. A comparison is made between four different adaptive algorithms for training time-series prediction ANNs in analyzing optimized training and potential errors. A hybrid algorithm combining Bayesian regularization with conjugate-gradient backpropagation is demonstrated to give the best average prediction accuracy, whilst a generalized regression $\mathrm{NN}$ is shown to reduce the possibility of isolated large prediction errors.

In Bathen et al [N30], multivariate models are proposed for the prediction of histological grade, hormone status, and axillary lymphatic spread in breast cancer patients. The multivariate methods applied are variable reduction by principal component analysis (PCA), and modelling by probabilistic neural network (PNN). Finally, the model is verified using prediction of blind samples. The verification results show that hormone status is well predicted by both PNN and PLS (partial least-squares regression) as a supplement for future clinical decision-making-concerning adjuvant treatment and the adaptation to more individualised treatment protocols.

\subsection{Other Applications}

Apart from the above four categories of applications, ANN has been applied to medical image processing for other purposes. Wu et al. [44] presents a new method to extract the patient information number (PIN) field automatically from the film-scanned image using a multilayer cluster neural network. Cerveri et al. [45] presented a hierarchical radial basis function (HRBF) network to correct geometric distortions in X-ray image intensifier, which reduces the accuracy of image-guided procedures and quantitative image reconstructions.

Hsu et al. [46] establish a method to predict and create surface a profile of bone defects by a well-trained 3-D orthogonal neural network. The coordinates of the skeletal positions around the boundary of bone defects are input into the 3-D orthogonal neural network to train it to team the scattering characteristic. The 3-D orthogonal neural network avoids local minima and converges rapidly. After the neural network has been well trained, the mathematic model of the bone defect surface is generated, and the pixel positions are derived.

It is difficult to generalise all these applications of ANN into to several united models. However, it might be possible to analysis the general pattern of applying ANNs. In the next Section, a comparison is made by studying the applications as described in all previous Sections. 


\section{Discussions and Conclusions}

As described in the previous five sections, applications of neural networks have been classified into four major categories. These applications seem quite different from each other and cover many aspects of medical image processing. The various different architectures available for medical imaging problems can present a dilemma for a prospective user. There are no rules or defined criteria that can be used to select the best network type, though the authors are confident that the examples presented throughout this paper will offer rules-of-thumb and guided inspiration for future efforts. To this end, all the neural networks successfully applied to medical imaging are highlighted and compared based on their application patterns, structures, operations, and training design etc. in Table 1. Since there is no theory to indicate what is the best neural network structure for medical image processing and pattern recognition, the information such as "Type of Network", "Type of input", "Number of Inputs", "Neurons in Hidden" and "Neurons in Output" is listed to help with searching and designing similar neural networks for the future applications. Although these applications may come from different areas such as CAD and segmentation, and inputs for neural networks are various, the essential purpose of applying these neural networks lies in their classifications, providing inspiring summary for existing modes of neural network applications and thus leading to further developments. Since the dataset for these applications are quite different, it is not possible to compare their results and the performance of these algorithms. Some applications are ignored in the list since the details about their neural networks are limited. The total number of neurons needed in the hidden layers somewhat depends on the total number of training samples.

Table 1 Comparative summary of feed-forward neural network applications in medical imaging

\begin{tabular}{|c|c|c|c|c|c|c|c|}
\hline Source & $\begin{array}{r}\text { Type of } \\
\text { Network }\end{array}$ & Purpose & $\begin{array}{l}\text { Type of } \\
\text { Input }\end{array}$ & $\begin{array}{l}\text { Number } \\
\text { of Inputs }\end{array}$ & $\begin{array}{l}\text { Neurons } \\
\text { in Hidden } \\
\text { layers }\end{array}$ & $\begin{array}{l}\text { Neurons } \\
\text { in Output }\end{array}$ & $\begin{array}{l}\text { Train/Test } \\
\text { /validation }\end{array}$ \\
\hline$[1]$ & $\begin{array}{l}\mathrm{CNN}^{*} \\
\left(\mathrm{BP}^{*}\right)\end{array}$ & Detect FP & $\begin{array}{l}\text { Intensity of } \\
\text { pixels }\end{array}$ & 256 & $14 / 10$ & 1 & $\begin{array}{c}268 \mathrm{ROI}^{*} / \\
267 \mathrm{ROI}\end{array}$ \\
\hline [3] & $\mathrm{BP}$ & $\begin{array}{l}\text { Reduce } \\
\text { FP }\end{array}$ & $\begin{array}{l}\text { Value of } \\
\text { features }\end{array}$ & 5 & 5 & 1 & $\begin{array}{l}1448 \text { clusters/ } \\
\text { leave-one-out }\end{array}$ \\
\hline [4] & MLP $^{*}$ & $\begin{array}{l}\text { Reduce } \\
\text { FP }\end{array}$ & $\begin{array}{l}\text { Value of } \\
\text { features }\end{array}$ & 9 & $20 / 10$ & 1 & Unknown \\
\hline [5] & $\mathrm{RBFNN}^{*}$ & $\begin{array}{l}\text { Classify } \\
\text { tissues }\end{array}$ & $\begin{array}{l}\text { Value of } \\
\text { features }\end{array}$ & 4 & 5 & 2 & $\begin{array}{l}44 \text { regions } \\
/ 54 \text { images }\end{array}$ \\
\hline [8] & $\mathrm{BP}$ & Detect FP & $\begin{array}{l}\text { Value of } \\
\text { features }\end{array}$ & 11 & 9 & 1 & $\begin{array}{l}100 \text { images / } \\
100 \text { images/ } \\
\text { Jackknife[47] }\end{array}$ \\
\hline [10] & BP & Detect FP & $\begin{array}{l}\text { Value of } \\
\text { features }\end{array}$ & 10 & 5 & 1 & $\begin{array}{c}\text { 397ROI/397 } \\
\text { ROI/Jackknife }\end{array}$ \\
\hline \multirow[t]{2}{*}{ [12] } & \multirow[t]{2}{*}{$\begin{array}{l}\text { Feed- } \\
\text { forward }\end{array}$} & $\begin{array}{l}\text { Classify } \\
\text { boundary }\end{array}$ & $\begin{array}{l}\text { Coordinate } \\
\text { /magnitude }\end{array}$ & 3 & $30 / 10$ & 1 & \multirow{2}{*}{$\begin{array}{l}100 \text { images/ } \\
147 \text { images \& } \\
65 \text { image CV }\end{array}$} \\
\hline & & $\begin{array}{l}\text { Classify } \\
\text { region }\end{array}$ & $\begin{array}{l}\text { Coordinate } \\
\text { /intensity }\end{array}$ & 3 & 50 & 1 & \\
\hline [14] & $\mathrm{BP}$ & Predict & Value of & 8 & 5 & 1 & 262/leave-one- \\
\hline
\end{tabular}




\begin{tabular}{|c|c|c|c|c|c|c|c|}
\hline & & tissue & features & 7 & 3 & 1 & out/Jackknife \\
\hline$[15]$ & BP & $\begin{array}{l}\text { Classify } \\
\text { tissues }\end{array}$ & $\begin{array}{l}\text { Value of } \\
\text { features }\end{array}$ & 3 & 10 & 1 & $\begin{array}{l}60 \text { primitives } \\
/ 983 \text { primitives }\end{array}$ \\
\hline$[22]$ & MLP & $\begin{array}{l}\text { Classify } \\
\text { boundary }\end{array}$ & $\begin{array}{l}\text { Intensity of } \\
\text { pixels }\end{array}$ & 49 & 30 & 1 & $\begin{array}{l}1200 \text { patterns / } \\
400 \text { slices }\end{array}$ \\
\hline [20] & BP & $\begin{array}{l}\text { Classify } \\
\text { tissues }\end{array}$ & $\begin{array}{c}\text { Statistical } \\
\text { indexes }\end{array}$ & 3 & Unknown & 3 & $\begin{array}{l}\text { Small number, } \\
\text { improved by } \\
\text { interaction }\end{array}$ \\
\hline [38] & BP & $\begin{array}{l}\text { Remove } \\
\text { noise }\end{array}$ & $\begin{array}{l}\text { Intensity of } \\
\text { pixels }\end{array}$ & 25 & 20 & 1 & Unknown \\
\hline [39] & $\begin{array}{l}\text { MTANN } \\
{ }_{*} \text { (BP) }\end{array}$ & $\begin{array}{c}\text { Classify } \\
\text { tissues }\end{array}$ & $\begin{array}{l}\text { Intensity of } \\
\text { pixels }\end{array}$ & 81 & 20 & 1 & $\begin{array}{l}5000 \text { Regions } \\
/ 118 \text { images }\end{array}$ \\
\hline [48] & $\mathrm{BP}$ & $\begin{array}{c}\text { Classify } \\
\text { MC }\end{array}$ & $\begin{array}{l}\text { Value of } \\
\text { features }\end{array}$ & 14 & 13 & 1 & $\begin{array}{c}100 \mathrm{ROI} / \\
\text { leave-one-out }\end{array}$ \\
\hline [49] & $\begin{array}{l}\text { MLBNN } \\
{ }^{*}(\mathrm{BP})\end{array}$ & $\begin{array}{c}\text { Classify } \\
\text { MC }\end{array}$ & $\begin{array}{l}\text { Vectors } \\
\text { from SOM }\end{array}$ & 5 & $25 / 14$ & 7 & $\begin{array}{l}32 \text { cases/ } 64 \\
\text { cases }\end{array}$ \\
\hline$[50]$ & $\mathrm{BP}$ & $\begin{array}{c}\text { Classify } \\
\text { tissues }\end{array}$ & $\begin{array}{c}\text { Vectors } \\
\text { from SOM }\end{array}$ & 3 & 7 & 7 & $\begin{array}{c}\text { Unknown/ } 80 \\
\text { images }\end{array}$ \\
\hline [51] & BP & $\begin{array}{l}\text { Detect } \\
\text { Edge }\end{array}$ & $\begin{array}{l}\text { Intensity of } \\
\text { pixels }\end{array}$ & 121 & 20 & 1 & $\begin{array}{l}24 \text { images/ } \\
\text { fourfold CV }\end{array}$ \\
\hline [N10] & BP & $\begin{array}{l}\text { Classify \& } \\
\text { evaluation }\end{array}$ & $\begin{array}{l}\text { Clinic and } \\
\text { radiological } \\
\text { findings }\end{array}$ & 15 & 9 & 4 & $\begin{array}{l}\text { MR images of } \\
126 \text { cases, } \\
\text { leave-one-out }\end{array}$ \\
\hline [N18] & $\begin{array}{c}\text { MLP } \\
\text { /RBFNN }\end{array}$ & $\begin{array}{l}\text { Detect } \\
\text { MCCs }\end{array}$ & intensity & 5 & 10 & 1 & $\begin{array}{c}\begin{array}{c}107 \mathrm{RIO} / 19 \\
\text { images? }\end{array} \\
\end{array}$ \\
\hline \multicolumn{5}{|c|}{$\begin{array}{ll}\text { - } & \text { BP: Back-propagation (feed forward) } \\
\text { - } & \text { CNN: Convolution neural network } \\
\text { - } & \text { CV: Cross validation } \\
\text { - } & \text { FP: False positive MC or regions } \\
\text { - } & \text { MC: Microcalcification cluster } \\
\text { - } & \text { MLBNN: Multi-layered BP neural network }\end{array}$} & \multicolumn{3}{|c|}{$\begin{array}{l}\text { MLP: Multiplayer perceptron } \\
\text { RBFNN: Radial basis function neural } \\
\text { network } \\
\text { ROI: Region of interest } \\
\text { SOM: Self-organizing map } \\
\text { MTANN: Massive training artificial neural } \\
\text { network }\end{array}$} \\
\hline
\end{tabular}

In contrast to feed forward neural network, the applications of feedback neural networks for medical image processing are quite limited in the past decade and most of them are in the area of image segmentation, which are primarily based on Hopfield neural networks. The similarities between these applications are quite limited but all of them need to minimise an energy function during convergence of the network. The energy function has to be designed individually, which might affect its application in medical imaging. Since the Hopfield neural network is unsupervised, it may not work for CAD like feed forward neural network that requires priori knowledge in classifications.

Although the applications of Kohonen's SOM are not as many as those of feed forward neural networks, its clustering and unsupervised properties make it very suitable for image registration. SOM converges to a solution that approximates its input data by adapting to prototype vectors. During this process, the relation of its neighbourhood neurons is also taken into account, leading to preservation of topology and mapping of training sets. This makes them particularly suitable for applications where dimensionality reduction is desirable and an output that can be easily interpreted is a necessary feature. In this sense SOMs may be more suitable for certain applications than other neural network architectures, and other pattern recognition and classification approaches. 
For the applications of image registration, the input vectors of the neurons in SOM usually contain the spatial coordinate and intensity of pixels. For applications in image compression, SOM is used as a topology preserving feature map to generate vector quantization for code words. Sometimes, SOM produces the segmentation results for feed forward neural networks due to its unsupervised clustering property.

In summary, the applications of ANN in medical image processing have to be analysed individually although many successful models have been reported in the literature. ANN has been applied to medical images to deal with the issues that can not be addressed by traditional image processing algorithms or by other classification techniques. By introducing artificial neural networks, algorithms developed for medical image processing and analysis often become more intelligent than conventional techniques. While this paper provided a focused survey on a range of neural networks and their applications to medical imaging, the main purpose here is to inspire further research and development on new applications and new concepts in exploiting neural networks.

While neural networks are undoubtedly powerful tools for classification, clustering and pattern recognition there are potential disadvantages when applying them to a given problem. Neural networks are notoriously hard to interpret and analyse, and in situations where it is desirable to simply and concisely define the process transforming inputs to output values it can be difficult to justify their use. While analysis of the internal weight and bias values for neurons in a network is possible, and a network itself can be represented formulaically, they are usually too large to be explained in a way that a human can easily understand. Despite this, they are still widely used in situations where a black-box solution is acceptable, and where experimental evidence of their accuracy is sufficient for testing and validation.

When compared to other machine learning approaches neural networks have many positive characteristics that must be considered by a prospective user. The variety of different network architectures and learning paradigms available, coupled with a theoretically limitless number of combinations of layers amounts, connections topologies, transfer functions and neuron amounts, make ANNs incredibly flexible processing tools. They can be applied to data with almost any number of inputs and outputs, and are well supported in different programming languages and software suites. Through manual modification of weights prior to training, and through imposing custom limitations on their modification during training, existing expert knowledge can be incorporated into their design and construction. Additionally, neural networks are usually computationally inexpensive to use after they have been trained, making them ideal for real-time applications where immediate output is desirable.

Recent results suggests they can still generate comparable results to state-of-art classifiers like SVM [N7]

Although this paper focuses on the various types of neural networks and how they can be applied to medical imaging, there are a variety of other approaches available for such an application. There are no clear 
rules or procedures that can be followed to determine if using a neural network is the best choice for a specific imaging problem, though guidance can be laid out to assist those that might consider their use. Neural networks undoubtedly possess strong potential for accurate output prediction, data clustering and topography-based mapping as can be seen by their widespread use in almost every discipline involving modelling and prediction. However, as discussed above, their inherent complexity makes them generally unsuitable for applications where post-training analysis of the way outputs are formed is necessary. In these situations there are clearly better choices of algorithm, such as decision trees, rule induction or Bayesian Networks where the impact that each input has upon the final result can bee seen more clearly, and often in an inherently human-understandable way.

Finally, the authors wish to acknowledge the financial support from the Breast Cancer Research Trust, in which the survey is carried out as part of the funded research project. 


\section{Reference:}

1 J. Ge, B. Sahiner, L. M. Hadjiiski, H. P. Chan, J. Wei, M. A. Helvie, and C. Zhou, "Computer aided detection of clusters of microcalcifications on full field digital mammograms," Medical Physics 33(8), pp: 2975-2988, 2006.

2 S. C. B. Lo, H. P. Chan, J. S. Lin, H. Li, M. T. Freedman and S. K. Mun, "Artificial convolution neural network for medical image pattern recognition", Neural networks, 8(7-8), pp. 1201-11214, 1995

3 R. H. Nagel, R. M. Nishikawa, J. Papaioannou, and K. Doi, "Analysis of methods for reducing false positives in the automated detection of clustered microcalcifications in mammograms," Medical Physics, 25(8), pp:1502-1506, 1998.

4 A. Papadopoulossa, D.I. Fotiadisb, A. Likasb, “An Automatic Microcalcification Detection System Based on a Hybrid Neural Network Classifier", Artificial Intelligence in Medicine, pp: 149-167, v.25, 2002.

5 I. Christoyiani, E. Dermatas and G. Kokkinakis, "Fast Detection of Masses in Computer Aided Mammography", IEEE Signal Processing Magazine, 17 (1), pp: 54-64, 2000.

6 A.C. Patrocinio, H. Schiabel, R.H. Benatti, C.E. Goes, and F.L.S. Nunes, "Investigation of clustered microcalcification features for an automated classifier as part of a mammography CAD scheme," Proc. the $22^{\text {nd }}$ Annual EMBS International Conf., July 23-28, 2000, pp. 12031205.

7 R. Setiono, "Extracting Rules from Pruned Neural Networks for Breast Cancer Diagnosis", Artificial Intelligence in Medicine, 8(1), pp: 37-51, 1996.

8 X. W. Xu, K. Doi, T. Kobayashi, H. MacMahon, and M. L. Giger, "Development of an improved CAD scheme for automated detection of lung nodules in digital chest images," Medical Physics, 24(9), pp.1395-1403, 1997.

9 Z. H. Zhou, Y. Jiang, Y. B. Yang and S. F. Chen, "Lung cancer cell identification based on artificial neural network ensembles," Artificial Intelligence in Medicine, 24(1), pp:25-36, 2002.

$10 \mathrm{~B}$ Keserci and $\mathrm{H}$ Yoshida, "Computerized detection of pulmonary nodules in chest radiographs based on morphological features and wavelet snake model," Medical Image Analysis 6(4), pp:431-447, 2002.

11 Qian W, Zhukov T, Song DS and Tockman MS, "Computerized analysis of cellular features and biomarkers for cytologic diagnosis of early lung cancer," Analytical and Quantitative Cytology and Histology, 29(2), pp:103-111, 2007.

12 G. Coppini, S. Diciotti, M. Falchini, N. Villari and G. Valli, "Neural networks for computeraided diagnosis: Detection of lung nodules in chest radiograms," IEEE Trans. Information Technology in Biomedicine, 7(4), pp: 344-357, 2003.

13 E. I. Mohamed, C. Maiolo, R. Linder, S. J. Poppl, and A. De Lorenzo, "Artificial neural network analysis: a novel application for predicting site-specific bone mineral density," Acta Diabetologica, 40, pp: S19-S22, Suppl. 1, 2003.

14 J. A. Scott, "The lung scan and the abnormal chest X-ray: difficult diagnoses," Nuclear Medicine Communications, 25(11), pp:1137-1141, 2004.

15 S. Kobashi, N. Kamiura, Y. Hata, and F. Miyawaki, "Volume-quantization-based neural network approach to 3D MR angiography image segmentation," Image and Vision Computing, 19(4), pp:185-193, 2001.

16 I. Middleton and R. I. Damper, "Segmentation of magnetic resonance images using a combination of neural networks and active contour models," Medical Engineering \& Physics, 26(1), pp:71-86, 2004.

17 J. S. Lin, "Segmentation of medical images through a penalized fuzzy Hopfield network with moments preservation," Journal of The Chinese Institute of Engineers, 23(5), pp:633-643, 2000 . 
18 K. C. R. Lin, M. S. Yang, H. C. Liu, J. F. Lirng and P. N. Wang, "Generalized Kohonen's competitive learning algorithms for ophthalmological MR image segmentation," Magnetic Resonance Imaging, 21(8), pp:863-870, 2003

19 Z. Dokur and T. Olmez, "Segmentation of MR and CT images by using a quantiser neural network, Neural Computing \& Applications," 11(3-4), pp: 168-177, 2003.

20 G. Stalidis, N. Maglaveras, S. N. Efstratiadis, A. S. Dimitriadis and C. Pappas, "Model-based processing scheme for quantitative 4-D cardiac MRI analysis," IEEE Trans. Information Technology In Biomedicine, 6(1), pp:59-72, 2002.

21 C. L. Chang and Y. T. Ching, "Fuzzy Hopfield neural network with fixed weight for medical image segmentation," Optical Engineering, 41(2), pp:351-358, 2002.

22 S. Shen, W. Sandham, M. Granat, and A. Sterr, "MRI fuzzy segmentation of brain tissue using neighborhood attraction with neural-network optimization," IEEE Trans. Information Technology In Biomedicine 9(3), pp:459-467, 2005.

23 C. Y. Chang and P. C. Chung, "Two-layer competitive based Hopfield neural network for medical image edge detection," Optical Engineering, 39(3), pp:695-703, 2000.

24 C. Y. Chang and P. C. Chung, "Medical image segmentation using a contextual-constraintbased Hopfield neural cube," Image and Vision Computing, 19(9-10), pp:669-678, 2001.

25 C. Y. Chang, "Contextual-based Hopfield neural network for medical image edge detection," Optical Engineering, 45(3), Art. No. 037006, 2006.

26 G. K. Matsopoulosa, N. A. Mouravlianskya, P. A. Asvestasa, K. K. Delibasisa and V. Koulouliasb, "Thoracic non-rigid registration combining self-organizing maps and radial basis functions," Medical Image Analysis, 9(3), pp. 237-254, 2005.

27 B. Fritzke, "A growing neural gas network learns topologies," Advances in Neural Information Processing Systems 7, MIT press, Cambridge, MA, pp. 625-632.

28 L. F. Shang, J. C. Lv, and Z. Yi, "Rigid medical image registration using PCA neural network," Neurocomputing, 69 (13-15), pp.1717-1722, 2006.

29 G. Coppini, S. Diciotti and G. Valli, "Matching of medical images by self-organizing neural networks," Pattern Recognition Letters 25(3), pp.341-352, 2004.

30 E. Fatemizadeh, C. Lucas and H. Soltanian-Zadeh, "Automatic landmark extraction from image data using modified growing neural gas network," IEEE Trans. Information Technology in Biomedicine, 7(2), pp:77-85, 2003.

31 S. Di Bona and O. Salvetti, "Neural method for three-dimensional image matching," Journal of Electronic Imaging 11(4), pp:497-506, 2002.

32 J. Jiang, "Image compression with neural networks - A survey," Image Communication, Journal of EURASIP, ELSEVIER, 14 (9), 1999.

33 N. G. Panagiotidis, D. Kalogeras, S. D. Kollias and A. Stafylopatis, "Neural network-assisted effective lossy compression of medical images," Proceedings of the IEEE, 84(10), pp:1474 1487, 1996.

34 B Karlik, "Medical image compression by using vector quantization neural network (VQNN)," Neural Network World, 16 (4), pp.341-348 2006.

35 A. Meyer-Base, K. Jancke, A. Wismuller, S. Foo and T. Martinetz, "Medical image compression using topology-preserving neural networks," Engineering Applications of Artificial Intelligence, 18(4), pp.383-392, 2005.

36 R. R. Jaiswal and A. N. Gaikwad, "Neural network assisted effective lossy compression of medical images," IETE TECHNICAL REVIEW, 23 (2): 119-126, 2006.

37 S. C. B. Lo, H. Li, M. T. Freedman, "Optimization of wavelet decomposition for image compression and feature preservation," IEEE Trans. Medical Imaging, 22(9), pp:1141-1151, 2003.

38 K. Suzuki, I. Horiba and N. Sugie, "Efficient Approximation of Neural Filters for Removing Quantum Noise from Images," IEEE Trans. Signal Processing, 50(7), pp:1787-1799, 2002. 
39 K. Suzuki, H. Abe, H. MacMahon and K. Doi, "Image-Processing Technique for Suppressing Ribs in Chest Radiographs by Means of Massive Training Artificial Neural Network (MTANN)," IEEE Trans. Medical Imaging, 25(4), 2006

40 K. Suzuki, I. Horiba, K. Ikegaya, and M. Nanki, "Recognition of coronary arterial stenosis using neural network on DSA system," Syst. Comput. Japan, vol. 26, no. 8, pp. 66-74, 1995.

41 K. Suzuki, "Neural edge enhancer for supervised edge enhancement from noisy images," IEEE Trans. Pattern Anal. Mach. Intell., vol. 25, no. 12,pp. 1582-1596, Dec. 2003.

42 L. Hainc and J. Kukal, "Role of robust processing in ANN de-noising of 2D image," Neural Network World, 16(2), pp:163-176, 2006.

43 G. K. Chen, T. D. Chiueh, J. H. Chen, "Active cancellation system of acoustic noise in MR imaging," IEEE Transactions On Biomedical Engineering, 46(2), pp:186-191, 1999.

$44 \mathrm{H}$. H. P. Wu, "Patient information extraction in digitized X-ray imagery," Image and Vision Computing 22(3), pp:215-226, 2004.

45 P. Cerveri, C. Forlani, N. A. Borghese and G. Ferrigno, "Distortion correction for x-ray image intensifiers: Local unwarping polynomials and RBF neural networks," Medical Physics 29(8), pp:1759-1771, 2002.

46 J. H. Hsu and C. S. Tseng, "Application of three-dimensional orthogonal neural network to craniomaxillary reconstruction," Computerized Medical Imaging and Graphics 25(6), pp:477482, 2001.

47 D.D. Dorfman, K.S. Berbaum and C.E. Metz, "Receiver operating characteristic rating analysis - generalization to the population of readers and patients with the jackknife method," Investigative Radiology, 27(9), pp: 723-731, 1992.

48 M. Kallergi, "Computer-aided diagnosis of mammographic microcalcification clusters," 31(2), Medical Physics, pp.314-326, 2004.

49 J.O. Glass, W. E. Reddick, O. Goloubeva, V. Yo and R.G. Steen, "Hybrid artificial neural network segmentation of precise and accurate inversion recovery (PAIR) images from normal human brain," MAGNETIC RESONANCE IMAGING, 18(10), pp:1245-1253, 2000.

50 W.E. Reddick, R.K. Mulhern, T.D. Elkin, J. O. Glass, T. E. Merchant and J. W. Langston, “A hybrid neural network analysis of subtle brain volume differences in children surviving brain tumors," MAGNETIC RESONANCE IMAGING, 16(4), pp.413-421, 1998.

51 K. Suzuki, I. Horiba, N. Sugie and M. Nanki, "Extraction of Left Ventricular Contours from Left Ventriculograms by Means of a Neural Edge Detector," IEEE Trans. Medical Imaging, 23(3), pp.330-339, 2004.

N12: J.-S. Chiu, Y.-F. Wang, Y.-C. Su, et al, "Artificial Neural Network to Predict Skeletal Metastasis in Patients with Prostate Cancer," Journal of Medical Systems, 33(2), pp. 91-100, April 2009.

N16: J. H. Goodband, O. C. L. Haas, and J. A. Mills, "A comparison of neural network approaches for on-line prediction in IGRT," Med. Phys., 35(3), pp. 1113-1122, March 2008.

N1: J. Kim and B. Horwitz, "Investigating the neural basis for fMRI-based functional connectivity in a blocked design: application to interregional correlations and psychophysiological interactions," Magn Reson Imaging, 26(5), pp. 583-593, 2008.

N6: G. Marrelec, J. Kim, J. Doyon, and B. Horwitz, "Large-Scale Neural Model Validation of Partial Correlation Analysis for Effective Connectivity Investigation in Functional MRI," Human Brain Mapping, 30(3), pp. 941-950, 2009.

N23: S. J. Carrington and A. J. Bailey, "Are there theory of mind regions in the brain? A review of the neuroimaging literature," Human Brain Mapping, 30(8), pp. 2313-2335, 2008. 
N34: K. Li, L. Guo, J. Nie and T. Liu, "Review of methods for functional brain connectivity detection using fMRI," Computerized Medical Imaging and Graphics, 33(2), pp. 131-139, 2009.

N28: H. D. Cheng, X. Cai, X. Chen, L. Hu, and X. Lou, "Computer-aided detection and classification of microcalcifications in mammograms: a survey," Pattern Recog., 36(12), pp. 2967-2991, 2003.

N29: H. D. Cheng, X. J. Shi, R. Min, L. M. Hu, X. P. Cai, and H. N. Du, "Approaches for automated detection and classification of masses in mammograms," Pattern Recog., 39(4), pp. 646-668, 2006.

N17: B. Verma, P. McLeod and A. Klevansky, "A novel soft cluster neural network for the classification of suspicious areas in digital mammograms," Pattern Recog., 42(9), pp. 1845$1852,2009$.

N18: S. Halkiotis, T. Botsis and M. Rangoussi, "Automatic detection of clustered microcalcifications in digital mammograms using mathematical morphology and neural networks," Signal Processing, 87(7), pp. 1559-1568, 2007.

N2: X. Zhang, M. Kanematsu, H. Fujita, et al, "Application of an artificial neural network to the computer-aided differentiation of focal liver disease in MR imaging," Radiological Physics and Technology, 2(2), pp. 175-182, 2009.

N8: D. Guo, T. Qiu, J. Bian, et al, "A computer-aided diagnostic system to discriminate SPIOenhanced magnetic resonance hepatocellular carcinoma by a neural network classifier," Computerized Medical Imaging and Graphics, 33(8), pp. 588-592, 2009.

N10: K. Yamashita, T. Yoshiura, H. Arimura, et al, "Performance Evaluation of Radiologists with Artificial Neural Network for Differential Diagnosis of Intra-Axial Cerebral Tumors on MR Images," American Journal of Neuroradiology, 29, pp.1153-1158, June-July 2008.

N11: F. Döhler, F. Mormann, B. Weber, et al, "A cellular neural network based method for classification of magnetic resonance images: Towards an automated detection of hippocampal sclerosis," Journal of Neuroscience Methods, 170(2), pp. 324-331, 2008.

N14: K. Tägil, J. Marving, M. Lomsky, et al, "Use of neural networks to improve quality control of interpretations in myocardial perfusion imaging," Int. J. Cardiovascular Imaging, 24(8), pp. 841-848, 2008.

N22: G. Pan, G. Yan, X. Song and X. Qiu, "BP neural network classification for bleeding detection in wireless capsule endoscopy," J. Medical Engineering and Techno., 33(7), pp. 575581, 2009.

N7: S.-T. Chen, Y.-H. Hsiao,, Y.-L. Huang et al, "Comparative Analysis of Logistic Regression, Support Vector Machine and Artificial Neural Network for the Differential Diagnosis of Benign and Malignant Solid Breast Tumors by the Use of Three-Dimensional Power Doppler Imaging," Korean J. Radiol., 10(5), pp. 464-471, 2009.

N3: M. A. Mazurowski, P. A. Habas, J. M. Zurada, et al, "Training neural network classifiers for medical decision making: The effects of imbalanced datasets on classification performance," Neural Networks, 21(2-3), pp. 427-436, 2008.

N4: P. J. Lisboa and A. F.G. Taktak, "The use of artificial neural networks in decision support in cancer: A systematic review," Neural Networks 19(4), pp. 408-415, 2006.

N9: K. Doi, "Computer-aided diagnosis in medical imaging: Historical review, current status and future potential," Computerized Medical Imaging and Graphics, 31(4-5), pp. 198-211, 2007.

N21: J. C. Fu, C. C. Chen, J.W. Chai, et al, "Image segmentation by EM-based adaptive pulse coupled neural networks in brain magnetic resonance imaging," Computerized Medical Imaging and Graphics, In Press, 2010.

N25: K. Suzuki, I. Horiba, N. Sugie, and M. Nanki, "Extraction of Left Ventricular Contours From Left Ventriculograms by Means of a Neural Edge Detector," IEEE Trans. Medical Imaging, 23(3), pp. 330-339, 2004. 
N24: V. E. Markaki, P. A. Asvestas, and G. K. Matsopoulos, "Application of Kohonen network for automatic point correspondence in 2D medical images," Computers in Biology and Medicine, 39(7), pp. 630-635, 2009.

N13: J. Zhang, Y. Ge, S. H. Ong, et al, "Rapid surface registration of 3D volumes using a neural network approach," Image and Vision Computing, 26(2), pp. 201-210, 2008.

N15: Z. Dokur, "A unified framework for image compression and segmentation by using an incremental neural network," Expert Systems and Applications, 34(1), pp. 611-619, 2008.

N31: A. E. Cavanna and M. R. Trimble, "The precuneus: a review of its functional anatomy and behavioural correlates,", Brain, 129(3), pp. 564-583, 2006.

N33: F. H. Guenther, S. S. Ghosh, and J. A. Tourville, "Neural modeling and imaging of the cortical interactions underlying syllable production," Brain and Language, 96(3), pp. 280-301, 2006.

N30: T. F. bather, L. R. Jensen, B. Sitter, et al, "MR-determined metabolic phenotype of breast cancer in prediction of lymphatic spread, grade, and hormone status," Breast Cancer Research and Treatment, 104(2), pp. 181-189, 2007. 